\title{
Novel Coronavirus (2019-nCoV) Outbreak- Break the Chain
}

\author{
Vidya Bhat S. ${ }^{1}$, Tasneem Shajahan ${ }^{2}$, Ramiz Ansari ${ }^{3}$, Sushanth S. Bhat ${ }^{4}$, \\ Sham S. Bhat ${ }^{5}$, Akhter Husain ${ }^{6}$, Laxmikanth Chatra $^{7}$
}

1Department of Prosthodontics and Crown and Bridge and Oral Implantology, Yenepoya Dental College, Mangalore, Karnataka, India. ${ }^{2}$ Department of Paedodontics and Preventive Dentistry, Yenepoya Dental College, Mangalore, Karnataka, India. ${ }^{3}$ Department of Orthodontics and Dentofacial Orthopaedics, Yenepoya Dental College, Mangalore, Karnataka, India. ${ }^{4}$ Department of Oral Medicine \& Radiology, Yenepoya Dental College, Mangalore, Karnataka, India.

${ }^{5}$ Department of Paedodontics and Preventive Dentistry, Yenepoya Dental College, Mangalore, Karnataka, India. ${ }^{6}$ Department of Orthodontics and Dentofacial Orthopaedics, Yenepoya Dental College, Mangalore, Karnataka, India.

${ }^{7}$ Department of Oral Medicine \& Radiology, Yenepoya Dental College, Mangalore, Karnataka, India.

\section{ABSTRACT}

\section{BACKGROUND}

Novel coronavirus has recently gained attention of the global community after it was first reported from Wuhan, China. It has been declared as global health emergency with implications to all people. Droplets of bodily fluids, such as saliva or mucus, from an infected person are dispersed in the air or on surfaces by coughing or sneezing and could be the main source for the further spread of infection. All healthcare professionals including Dental Surgeons should help to prevent transmission of this deadly virus. It's a virus affecting both human and animals and spreads from animals and person to person in close proximity, similar to other respiratory illnesses, such as the flu. This review article mainly highlights the types, epidemiology, transmission and addresses the implications of $2019 \mathrm{n}-\mathrm{CoV}$ from the health professionals perspective and discusses the necessary preventive precautions that should be strictly followed in a dental setup so as to prevent further spread of infection.

\section{KEY WORDS}

Corona Virus, Infection Control, Prevention, Dental Surgeon
Corresponding Author: Dr. Tasneem Shajahan Postgraduate Student, Department of Pedodontics and Preventive Dentistry, Yenepoya Dental College, Yenepoya University, Derlakatte, Mangalore-575018, Karnataka, India. E-mail: tasneemshajahan1994@gmail.com

DOI: $10.14260 /$ jemds/2020/342

Financial or Other Competing Interests: None.

How to Cite This Article:

Bhat VS, Bhat SS, Shajahan T, et al. Novel coronavirus (2019-nCoV) outbreak- break the chain. J. Evolution Med. Dent. Sci. 2020;9(19):1567-1571, 10.14260/jemds/2020/342

Submission 05-02-2020,

Peer Review 24-04-2020,

Acceptance 30-04-2020,

Published 11-05-2020. 


\section{BACKGROUND}

The World Health Organisation (WHO) declared the new coronavirus epidemic a Public Health Emergency of International Concern, or PHEIC after it was informed of cases of pneumonia of unknown aetiology detected in Wuhan City of China. A mysterious new strain of coronavirus has killed over 200 people in China and infected thousands, including in other countries. One positive case has been reported in India, where authorities are working to contain the spread of the pathogen, known as the 2019 Novel Coronavirus (2019-nCoV).

A corona virus are a large extended family of viruses that causes infection in nose, sinuses and upper throat. The name corona is derived from the Latin word corona and the Greek which means a crown or halo. They are so named due to their crown like shape characterized by fringes of large and bulbous surface projections creating an image of royal crown. This morphology is created by the viral spike (S) peplomers, which are proteins that populate the surface of the virus. They are transmitted mainly between animals and people, thereby making them zoonotic in nature.

\section{Human Coronaviruses}

Coronaviruses are important human and animal pathogens. During epidemics, they are the cause of up to one-third of community-acquired upper respiratory tract infections in adults which include common cold with major symptoms like fever, throat pain, swollen adenoids and rarer forms like severe acute respiratory tract infections (SARS) and middle east respiratory syndrome (MERS) which could be seriously fatal. In addition, certain coronaviruses are also known to cause diarrhoea in infants and children. ${ }^{1}$

The much-publicized human coronavirus discovered in 2003, SARS-CoV, which causes severe acute respiratory syndrome (SARS) affects both upper and lower respiratory tract, thereby making it unique from the remaining corona viruses.

Human coronaviruses accounts for five to ten per cent of all acute upper respiratory tract infections in adults and is known to cause mild flu-like illness to pneumonia-like presentations.

\section{KNOWN STRAINS OF HUMAN CORONA} VIRUSES ${ }^{2}$

\section{Human Coronavirus 229E (HCoV-229E)}

Human coronavirus 229E is a single-stranded, positive-sense, RNA virus species in the family Corona viridae. Along with Human coronavirus OC43, it is considered responsible for the common cold.

\section{Human Coronavirus OC43 (HCoV-OC43)}

Along with $\mathrm{HCoV}-229 \mathrm{E}$, they cause severe lower respiratory tract infections, including pneumonia in infants, the elderly, and immunocompromised individuals.

\section{SARS-CoV}

SARS-CoV often causes a severe illness marked initially by systemic symptoms of muscle pain, headache, and fever, followed in 2-14 days by the onset of respiratory symptoms, mainly cough, dyspnoea, pneumonia and decrease in the number of lymphocytes circulating in the blood.

\section{Human Coronavirus NL63 (HCoV-NL63, New Haven Coronavirus)}

Human coronavirus NL63 or HCoV-NL63 is a species of Alphacoronavirus that was identified in late 2004 in a seven-month-old child with bronchiolitis in the Netherlands.

\section{Human Coronavirus HKU1}

HCoV-HKU1 was first reported in January, 2005, in a 71-yearold man who was hospitalized with an acute respiratory distress and was radiographically confirmed bilateral pneumonia.

\section{Middle East Respiratory Syndrome Coronavirus (MERS-CoV)}

Previously known as novel coronavirus 2012 and HCoV-EMC. Novel coronavirus 2012 (2012-nCoV) or novel coronavirus (nCoV), was first reported in 2012 after genome sequencing of a virus isolated from sputum samples from a person who fell ill in a 2012 outbreak of a new flu.

\section{Novel Coronavirus (2019-nCoV) - (Wuhan Corona Virus)}

The novel coronavirus (2019-nCoV), informally known as the Wuhan coronavirus, first identified by authorities in Wuhan, Hubei, China, as the cause of the ongoing 2019-20 Wuhan coronavirus outbreak.

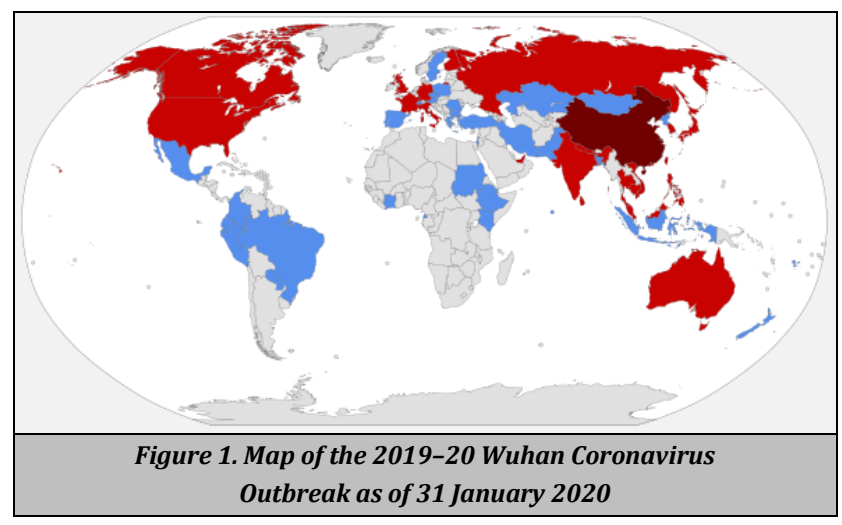




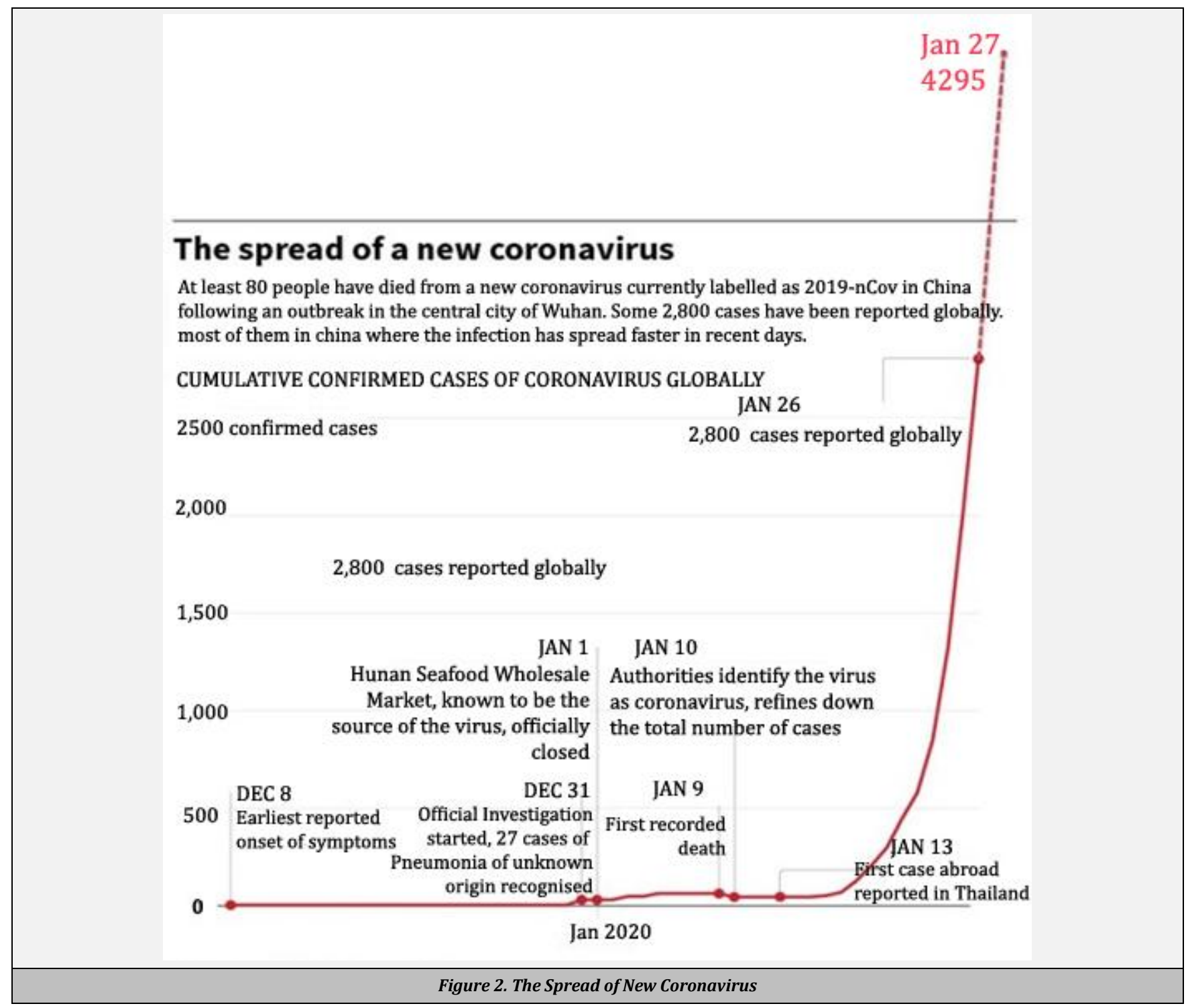

\section{Epidemiology}

An outbreak of 2019-nCoV was first detected in Wuhan, China, in mid-December 2019. Till date, more than 9500 cases of infection have been reported from China, and other cases were people who have travelled from Wuhan or were in direct contact with someone who travelled from the area. The number of deaths was 213 as of 31 January 2020. Human-tohuman spread was first confirmed in Guangdong, China, on 20 January 2020 and later been confirmed in Germany, Taiwan, Japan, the United States and the United Kingdom.

On 30 January 2020, 2019-nCoV was designated a global health emergency by the World Health Organization (WHO). The WHO has coined the interim term "2019-nCoV acute respiratory disease" to describe the disease caused by the virus. India confirmed its first positive case of novel coronavirus on 30th January 20202 (Figure 1) (Figure 2).

\section{Treatment for Corona Virus}

There is no vaccine or specific antiviral treatment as if now recommended for 2019-nCoV infection. People infected with 2019-nCoV should receive supportive care to help relieve symptoms. For severe cases, treatment should mainly emphasize on supporting vital organ functions. People who think they may have been exposed to 2019-nCoV should immediately contact their healthcare provider.

\section{PREVENTION IS BETTER THAN CURE- GENERAL RECOMMENDATIONS}

\section{WHO Recommendations ${ }^{3}$}

1. General hygiene measures, including regular hand washing with soap and potable water after touching animals and animal products, avoiding touching eyes, nose or mouth with hands and avoiding contact with sick animals or spoiled animal products should be practiced by all visiting animal markets. Any contact with animals living in the market (e.g., stray cats and dogs, rodents, birds, bats) should be strictly avoided. Avoid contact with potentially contaminated animal waste or fluids on the soil or structures of shops and market facilities.

2. Avoid consumption of raw or undercooked animal products. Raw meat, milk or animal organs should be 
handled with care, to avoid cross- contamination with uncooked foods, as per good food safety practices.

\section{Recommendations for At-Risk Groups}

- People with underlying medical conditions, considered as higher risk of severe disease, should avoid contact with live animal markets, stray animals and wild animals and should avoid eating raw meat.

- $\quad$ Good personal hygiene including frequent hand washing is considered mandatory for slaughterhouse workers, veterinarians, market workers after touching animals and animal products

- Wearing protective gowns, gloves, masks while professionally handling animals and animal products.

- Disinfection of equipment's and working stations at least once in a day.

- Protective clothing should be removed and washed every day.

- Protective clothes and items are recommended to remain in work place for daily washing which in turn protects the family members from getting exposed to soiled work clothing, shoes or other items that may have come into contact with potentially contaminated material.

- Avoid sick animals to be slaughtered for consumption and avoid contact with their fluids.

- Dead animals should be safely buried and destroyed.

\section{Personal Protection}

- Medical mask is one of the preventive measures to limit spread of certain respiratory diseases including 2019 nCoV.

- It is also recommended to follow hand hygiene and preventive measures to prevent transmission.

\section{COMMUNITY SETTING}

\section{Individuals without Respiratory Symptoms Should}

- Avoid groupings and frequency of closed crowded spaces.

- Maintain distance of at least 1 meter from any individual with 2019-nCoV respiratory symptoms (e.g., coughing, sneezing)

- $\quad$-Perform hand hygiene frequently, using alcohol based hand rub if hands are not visibly soiled or soap and water when hands are visibly soiled

- $\quad$ Cover nose and mouth with flexed elbow or paper tissue while coughing and sneezing and dispose the tissue immediately after use and follow performing hand hygiene measures.

- Refrain from touching mouth and nose.

- A medical mask is not required to protect non-sick persons. However if used, best practices should be followed on how to wear, remove and disposal followed by hand hygiene measures.

\section{Individuals with Respiratory Symptoms Should}

- Wear a medical mask and seek medical care if experiencing fever, cough and difficulty in breathing.
- WHO recommends that suspected cases of 2019-nCoV infection be cared for using isolation precautions and monitored in a hospital setting which would ensure both safety and quality of health care and public health security.

\section{Dental Perspective}

Medical professional which include Doctors, Dental surgeons, nurses and specialists are always at an increased risk of infection due to their constant close contact with various patients. The dental team may encounter patients who have recently flew from the affected areas. Individuals who have come from the affected area but are still asymptomatic and had no direct contact are considered to be at low risk and have no restrictions to medical and dental care. However, on the other hand the ones who may have had contact should have any nonessential treatment deferred.

\section{Patient Evaluation}

A sharp-eyed surveillance, prompt diagnosis and proper isolation will help to prevent the spread of the disease. The dental surgeons should be alert and should always promptly evaluate the patients who:

- Have travelled from countries in or near china and have developed severe acute respiratory illness.

- Are close contacts of a symptomatic recent traveller from the area who has fever and acute respiratory illness

- Are close contacts of a confirmed case.

\section{INFECTION CONTROL IN DENTAL CLINICS}

\section{Standard Precautions}

The dental surgeons should make sure to stick on to the standard infection control measures while managing patients under investigation or symptomatic patients.

- The dental treatment should be postponed if the patient is suspected to have the signs and symptoms of 2019 nCoV.

- Emergency dental treatment should be delivered in a room with proper ventilation and proper mask filtration.

- Prevention of needle sticks or sharp injuries.

- Perform Hand hygiene after coming contact with respiratory secretions.

- Advice patients to cover their nose while coughing and sneezing.

- Rational, correct and consistent use of personal protective equipment (PPE).

- Droplet precautions should be added to standard precautions.

- Eye protection and preventive measures should be considered.

- Wear a medical mask when entering a room where patients are suspected or confirmed of being infected with 2019-nCoV.

- Always follow the 5 moments for hand hygiene should include before touching a patient, before any clean or aseptic procedure, after body fluid exposure risk, after 
touching a patient, and after touching a patient's surroundings, including contaminated items or surfaces.

- Ensure that cleaning and disinfection procedures are followed consistently and correctly. Cleaning environmental surfaces with water and detergent and applying commonly used disinfectants (such as hypochlorite) is an effective and sufficient procedure.

\section{Masks Management ${ }^{5}$}

- Appropriate use and disposal of masks are highly essential to avoid the risk of transmission.

- The mask should be carefully and securely placed to minimise any gaps between the face and the mask.

- Avoid touching the mask when in use.

- Removal of the mask should be done appropriately (i.e. do not touch the front but remove the lace from behind)

- Do not re-use single use masks.

- Discard single use masks after each use and dispose immediately.

- If advertently the mask is being touched, clean hands by using an alcohol based hand rub or soap and water

II. Infection Prevention and Control Precautions for Aerosol-Generating Procedures

- The procedure performing room should be an adequately ventilated room and the specifications are natural ventilation with air flow of at least $60 \mathrm{~L} / \mathrm{s}$ per patient or in negative pressure rooms with at least 12 air changes per hour and controlled direction of air flow when using mechanical ventilation.

- The respirator used should be at least as protective as a US National Institute for Occupational Safety and health (NIOSH) certified N95, European Union (EU) standard FFP2 or equivalent

- Disposable respirator should always undergo the seal check.

- $\quad$ Always use eye protection (goggles or a face shield)

- Wear a clean, non-sterile, long sleeved gown and gloves.

- If gowns are not fluid resistant, use a waterproof apron for infection prevention and control.

- Limit the number of persons present in the room to the minimum required for patients care and support.

- Use single use disposable equipment.

III. Additional Infection Prevention and Control Precautions When Caring for Patients with Acute Respiratory Infection (ARI)

- Wear a medical mask when in close contact (i.e. within approximately $1 \mathrm{~m}$ ) with a patient with acute respiratory tract infection.

- Perform hand hygiene before and after contact with the patient and his or her surroundings and immediately after removal of a medical mask.
IV. Additional Infection Prevention and Control Precautions When Caring for Patients with Probable or Confirmed nCoV Infection

- Family members and visitors in contact with a patient should be limited to those essential for patient support and should be trained on the risk of transmission

- Notify the receiving area of the patient's diagnosis and necessary precautions as soon as possible before the patient's arrival.

- Clean and disinfect patient-contact surfaces (e.g. dental chair) after use.

- Refrain from touching eyes, nose or mouth with potentially contaminated hands.

\section{CONCLUSIONS}

Corona virus is a terrifying disease which could be fatal if left untreated. Even though the dental team is not at a high risk of coming in contact with the disease, it poses a high risk of transmission if undetected cases visit the clinic. The dental team should always be prepared to treat an undetected case by strictly following the infection control protocols in the dental clinic and should be aware of health protection measures against the virus.

Recommendations based on information available at the time of preparing the article.

\section{REFERENCES}

[1] Madani TA, Althaqafi AO, Alraddadi BM. Infection prevention and control guidelines for patients with Middle East respiratory syndrome coronavirus (MERSCoV) infection. Saudi Med J 2014;35(8):897-913.

[2] https://en.wikipedia.org/wiki/Coronavirus

[3] World Health Organization. Infection prevention and control of epidemic-and pandemic-prone acute respiratory infections in health care. World Health Organization, 2014.

[4] Infection prevention and control during health care when novel coronavirus (nCoV) infection is suspected Interim guidance 25 January 2020. WHO/2019$\mathrm{nCoV} / \mathrm{IPC} / \mathrm{v} 2020.2$

[5] Advice on the use of masks the community, during home care and in health care settings in the context of the novel coronavirus (2019-nCoV) outbreak interim guidance 29 January 2020. WHO/nCoV/IPC_Masks/2020. 